\title{
Analysis of geometrical effects on the behavior of transverse and longitudinal modes of amorphous silicon compounds
}

\author{
J. A. Moreno, B. Garrido, a) J. Samitier, and J. R. Morante \\ Electronic Materials \& Engineering laboratory (E.M.E.), Departament de Física Aplicada i Electrònica, \\ Universitat de Barcelona, Diagonal 645-647, 08028 Barcelona, Spain
}

(Received 24 June 1996; accepted for publication 12 November 1996)

\begin{abstract}
The peak frequency, width, and shape of the transverse-optical (TO) and longitudinal-optical (LO) infrared absorption modes of silicon oxides $\left(\mathrm{SiO}_{2}, \mathrm{SiO}_{x}\right)$, silicon nitrides $\left(\mathrm{Si}_{3} \mathrm{~N}_{4}, \mathrm{SiN}_{x}\right)$, silicon oxynitrides $\left(\mathrm{SiO}_{x} \mathrm{~N}_{y}\right)$, and other silicon compounds have often been connected with stress, stoichiometry, defects, structural order, and other properties of the layers. However, certain geometrical effects strongly influence the spectral response of the material independent of its physical or structural properties. The influence of layer thickness, multilayer configuration, substrate effects, angles, and polarization on the behavior of TO and LO bands are presented and discussed. Some corrections are suggested to reduce experimental error and for the reliable measurement of stress, composition, disorder, and structure. (C) 1997 American Institute of Physics.
\end{abstract}

[S0021-8979(97)05104-9]

\section{INTRODUCTION}

Infrared transmission spectroscopy is widely used to obtain information about the composition and structure of dielectrics and semiconductors. In the study of the vibrational spectra, band parameters of transmission or reflection resonances such as peak frequency, width, shape, and intensity are correlated with the physical and microstructural properties of the materials. For instance, in thin $\mathrm{SiO}_{2}$ films, intrinsic stresses are often measured from the peak frequency of the transverse-optical (TO) and longitudinal-optical (LO) stretching modes of the $\mathrm{Si}-\mathrm{O}$ bond $\left(\mathrm{TO}_{3}\right.$ and $\mathrm{LO}_{3}$, respectively) by using models applicable to the short-range order of the amorphous structure ${ }^{1-3}$ Moreover, the composition and microstructure of nonstoichiometric silicon oxides, nitrides, oxynitrides, and other silicon compounds are frequently measured from the peak frequency, shape, and other features of the infrared bands. ${ }^{4-19}$ Evaluation of the hydrogen content (or other impurities) in deposited or grown amorphous layers are routinely determined from the intensity and position of their respective infrared resonances. ${ }^{20-22}$

Spectral variations are almost always assumed to be due to variations in physical properties of the layers and less frequently in the thicknesses of the layers, angle, and polarization of incident light and multilayer arrangement. The results are usually coherent, especially when samples with similar thicknesses and in the same experimental conditions are compared. However, comparisons between the results obtained by different workers are always difficult because experimental values of peak frequencies, widths, and intensities of spectral bands are scattered over a wide range. A good illustration is provided by the study of stresses in thermal $\mathrm{SiO}_{2}$ layers. The peak frequency given for the $\mathrm{TO}_{3} \mathrm{ab}-$ sorption of $400 \AA$ thick layers after relaxation of the structure by annealing is around $1075 \mathrm{~cm}^{-1}$. However, for relaxed oxides of $1000 \AA$, in principle with the same structure, the values reported are around $1083-1084 \mathrm{~cm}^{-1}$; moreover, for

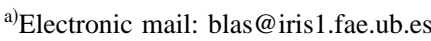

the same oxides but with thicknesses around 4000-5000 $\AA$, the values reported, always after annealing, are in the range between 1090 and $1095 \mathrm{~cm}^{-1}$. Obviously, there exist differencies between samples originated exclusively from a different structure (the oxide close to the interface is denser due to the presence of a compressive stress ${ }^{13,14}$ ), but one must be able to separate them from thickness and interference effects.

Some previous studies have reported variations in the peak parameters of the spectra with the thickness of the layers, mainly for $\mathrm{SiO}_{2} \cdot{ }^{7,23-26}$ In view of those particular modifications of the spectra, it is the purpose of this paper to quantify their magnitude and influence and to propose corrections to reduce their effects. The paper is organized as follows: First, we present a study of the bands generated with a computer simulation program that is based on the matrix formalism for field propagation through a multilayer system. Second, these results are compared with the experimental data obtained from the infrared analysis of thermal $\mathrm{SiO}_{2}$ layers with different thicknesses grown onto silicon wafers. Some results concerning silicon nitrides are also considered. Third, we propose an explanation of the origin of geometrical effects and the corrections that should be taken into account. Finally, the equations for the reflection and transmission coefficients on multilayer structures are developed in Taylor series with thickness as an independent variable to evaluate analytically the geometrical effects for thin layers.

\section{GEOMETRICAL EFFECTS IN LAYERED SYSTEMS: SIMULATION ANALYSIS}

For the optical study of multilayered materials we used the approach of Harbecke. ${ }^{27-30}$ This method allows calculation of the complex-amplitude reflection and transmission coefficients $(r$ and $t)$ for a pile of faced absorbent layers. The main goal of the procedure is that it is able to eliminate the interference fringes of thick layers from the concept of the coherent and incoherent multiple reflections within the layers. Thus, in the model calculations it was possible to avoid the very narrow interferences of the thick silicon substrate. 


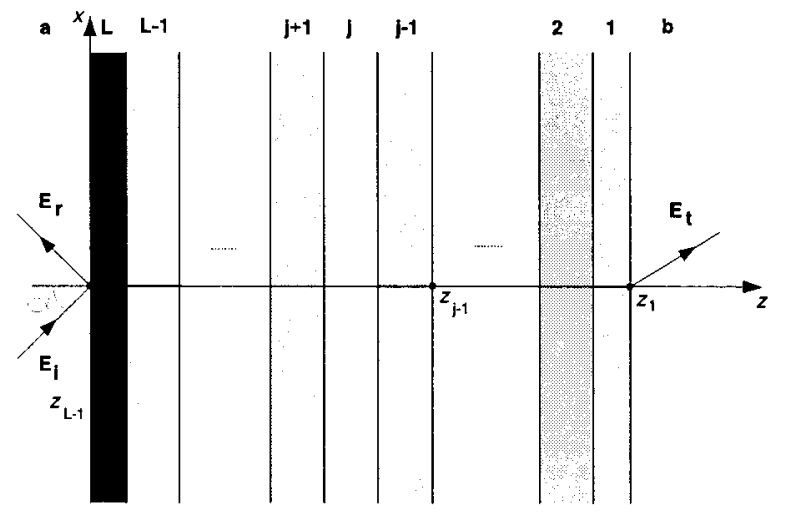

FIG. 1. Schematic representation of the model and coordinates used for the multilayered system.

The transformation of fields across the interface between two layers and the propagation inside each layer are considered from the well-known matrix formalism developed by Abelès. $^{27,28}$ If the multilayer consists of $L$ parallel layers (Fig. 1), the final transformation of fields from right to left is the result of the product of $2 L+1$ matrices, $L+1$ interfaces for passing $\left(\left[\mathbf{M}_{j}\right], j=1, \ldots, L+1\right)$, and $L$ matrices for in-layer propagation $\left(\left[\mathbf{P}_{j}\right], j=1, \ldots, L\right)$. In the case of $s$ polarization (transverse electric wave), we consider the propagation of the electric field $\mathbf{E}=(0, E, 0)$, which is parallel to the interfaces. For $p$ polarization (transverse magnetic wave), we consider the field $\mathbf{H}=(0, H, 0)$, now parallel to the interfaces. Then, for $s$ polarization, when an electromagnetic wave $E_{i}$ is projected on the pile, the reflected wave $E_{r}$ and the transmitted wave $E_{t}$ are connected with

$$
\begin{aligned}
\left(\begin{array}{c}
E_{i} \\
E_{r}
\end{array}\right)_{z=0^{-}} & =\left[\mathbf{M}_{L+1}\right]\left[\mathbf{P}_{L}\right]\left[\mathbf{M}_{L}\right] \cdots\left[\mathbf{P}_{1}\right]\left[\mathbf{M}_{1}\right]\left(\begin{array}{c}
E_{t} \\
0
\end{array}\right)_{z=z_{1}^{+}} \\
& \equiv\left(\begin{array}{ll}
T_{11} & T_{12} \\
T_{21} & T_{22}
\end{array}\right)\left(\begin{array}{c}
E_{t} \\
0
\end{array}\right)_{z=z_{1}^{+}} .
\end{aligned}
$$

The modifications for $p$ polarization and the detailed expressions of the transformation matrices are given in the Appendix, and include the Fresnel's coefficients, which depend on the complex dielectric functions $\epsilon_{j}$ of the materials. Thus, the complex-amplitude reflection and transmission coefficients of the whole pile are

$$
\begin{aligned}
& r=\frac{E_{r}}{E_{i}}=\frac{T_{21}}{T_{11}}, \\
& t=\frac{E_{t}}{E_{i}}=\frac{1}{T_{11}} .
\end{aligned}
$$

Finally, the reflection $(R)$ and transmission $(T)$ of the multilayer are given by

$$
\begin{aligned}
& R=r r^{*}, \\
& T=\frac{\operatorname{Re}\left[N_{b}\right]}{\cos \alpha} t t^{*},
\end{aligned}
$$

where $N_{b}$ is the generalized refractive index of the back media (defined in the Appendix) and $\alpha$ is the angle of incidence (Fig. 1).

The inputs to the simulation program were the complex dielectric functions $\epsilon_{j}(\omega)(j=1, \ldots, L)$ of each layer. The optical properties of the materials are embodied in $\epsilon(\omega)$ and its dependence on frequency. We assumed the materials to be composed of a set of dumped harmonic oscillators, one for each absorption mode. All the simulations were performed by using the results of two models for the frequency dependence of the dielectric function. (i) model 1 (three parameters) is based on oscillator terms of the following structure:

$$
\epsilon(\omega)=\epsilon_{\infty}+\sum_{k} \frac{4 \pi \Omega_{k}^{2}}{\omega_{0 k}^{2}-\omega^{2}-i \gamma_{k} \omega},
$$

where $k$ runs across the relevant vibration modes, $\Omega_{k}, \omega_{0 k}$, and $\gamma_{k}$ are, respectively, the oscillator strength, frequency of resonance, and dumping constant of the mode $k$, and $\epsilon_{\infty}$ represents the polarizability due to electronic resonances. (ii) model 2 (four parameters) has an additional parameter, $\sigma_{k}$, which accounts for the dispersion in frequency of the oscillator strength of each mode (the Gaussian distribution for symmetric peaks). The oscillator terms have the following expression:

$$
\begin{aligned}
\epsilon(\omega)= & \epsilon_{\infty} \\
& +\sum_{k} \frac{1}{\sigma_{k} \sqrt{\pi}} \int_{0}^{\infty} \frac{4 \pi \Omega_{k}^{2} \exp \left[-\left(\Psi-\omega_{0 k}\right)^{2} / \sigma_{k}^{2}\right]}{\Psi^{2}-\omega^{2}-i \gamma_{k} \omega} d \Psi .
\end{aligned}
$$

Model 2 has been reported to reproduce the spectra of amorphous materials such as $\mathrm{SiO}_{2}$ and $\mathrm{SiN}_{x}$ successfully.,

The two main options of the simulation program are (i) determination of dielectric functions from reflectance or transmittance measurements (by a fitting procedure) and (ii) simulation of reflection and transmission spectra for any multilayered system under various experimental conditions. $^{25,31}$ The results based on the model 1 and model 2 for thermally grown $\mathrm{SiO}_{2}$ and deposited $\mathrm{Si}_{3} \mathrm{~N}_{4}$ layers are shown in Table I. These results were the input data to all the simulations performed in the present work and agree with similar calculations reported in the literature. ${ }^{26}$

We simulated the reflection, transmission, and absorbance spectra of $\mathrm{SiO}_{2}$ and $\mathrm{Si}_{3} \mathrm{~N}_{4}$ layers grown or deposited onto silicon wafers. The absorbance spectra of some $\mathrm{SiO}_{2}$ layers are shown for illustration in Fig. 2. Briefly, the stretching band of $\mathrm{SiO}_{2}$ is composed of two TO modes: the $\mathrm{TO}_{3}$ mode is associated with the asymmetric stretching motion of the oxygens atoms of two adjacent $\mathrm{Si}-\mathrm{O}-\mathrm{Si}$ units, and its high-frequency shoulder $\left(\mathrm{TO}_{4}\right.$ mode), which has been attributed to the symmetric stretching motion of $\mathrm{Si}-\mathrm{O}-\mathrm{Si}$ adjacent units. The $\mathrm{TO}_{4}$ mode has been related to the disorder in the amorphous structure of $\mathrm{SiO}_{2}{ }^{5}$ In thermal oxides, these modes are centered, respectively, around 1070-1090 $\mathrm{cm}^{-1}$ and $1200 \mathrm{~cm}^{-1}$. We have also studied their related longitudinal modes $\mathrm{LO}_{3}\left(\sim 1256 \mathrm{~cm}^{-1}\right)$ and $\operatorname{LO}_{4}\left(\sim 1160 \mathrm{~cm}^{-1}\right)$, 
TABLE I. Parameters of the dielectric function for thermally grown $\mathrm{SiO}_{2}$ and deposited $\mathrm{Si}_{3} \mathrm{~N}_{4}$ layers. The values of model 2 for the oxide were obtained after fitting the experimental reflection and transmission spectra. The values of model 1 for the oxide and model 2 for the nitride were extracted from literature (Refs. 4 and 30). $\Omega, \omega_{0}$, and $\gamma$ are, respectively, the oscillator strength, frequency of resonance, and dumping constant of the different modes. Model 2 has an additional parameter, $\sigma$, which accounts for the dispersion in frequency of the oscillator strength of each mode. The best damping parameter for all the modes in model 2 was $8 \mathrm{~cm}^{-1}$.

\begin{tabular}{crcc}
\hline \hline Model 1 & $\Omega^{2}\left(\mathrm{~cm}^{-2}\right)$ & $\omega_{0}\left(\mathrm{~cm}^{-1}\right)$ & $\gamma\left(\mathrm{cm}^{-1}\right)$ \\
\hline Silicon oxide & 59818 & 1075 & 20 \\
& 5630 & 1190 & 70 \\
Model 2 & $\Omega^{2}\left(\mathrm{~cm}^{-2}\right)$ & $\omega_{0}\left(\mathrm{~cm}^{-1}\right)$ & $\sigma\left(\mathrm{cm}^{-1}\right)$ \\
\hline Silicon oxide & 40100 & 1067 & 30 \\
& 3900 & 1092 & 11 \\
& 3200 & 1164 & 30 \\
& 2200 & 1218 & 35 \\
& 4100 & 896 & 34 \\
& 7500 & 962 & 36 \\
& 12000 & 1026 & 34 \\
& 25200 & 1070 & 35 \\
& 3700 & 1159 & 32 \\
& 2300 & 1219 & 35 \\
\hline \hline
\end{tabular}

which are excited under oblique incidence by the component of the electric field perpendicular to the sample surface ( $p$ polarization). ${ }^{5,32}$

After baseline subtraction ${ }^{33}$ we determined the peak frequency, peak width, and intensity of the bands. We found large variation of these features with the thickness of the layer and angle of incidence of the radiation, though we did not vary the dielectric functions used as data for the simulation program. In Fig. 3 we show the variation of the $\mathrm{TO}_{3}$

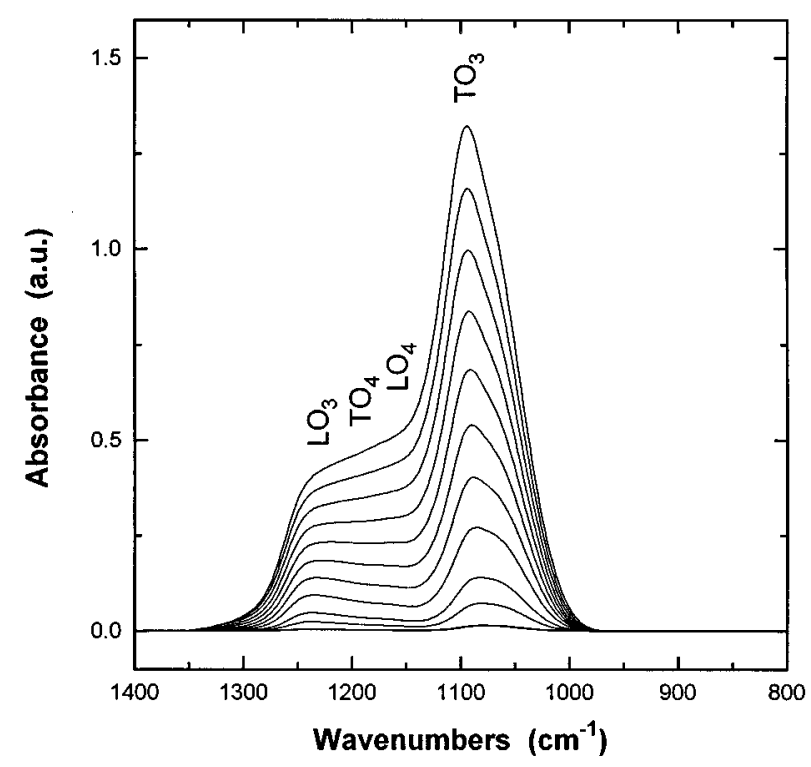

FIG. 2. Simulated absorbance at $45^{\circ}$ of incidence of $\mathrm{SiO}_{2}$ layers on $\mathrm{Si}$ substrate for different thicknesses (model 2). The intensity increases with the thickness of the layer $(100 \AA, 500 \AA$, and from 1000 to $9000 \AA$ in steps of $1000 \AA)$. The peak frequencies of the transverse optical modes $\left(\mathrm{TO}_{3}\right.$ and $\left.\mathrm{TO}_{4}\right)$ and the longitudinal optical modes $\left(\mathrm{LO}_{3}\right.$ and $\left.\mathrm{LO}_{4}\right)$ are indicated.

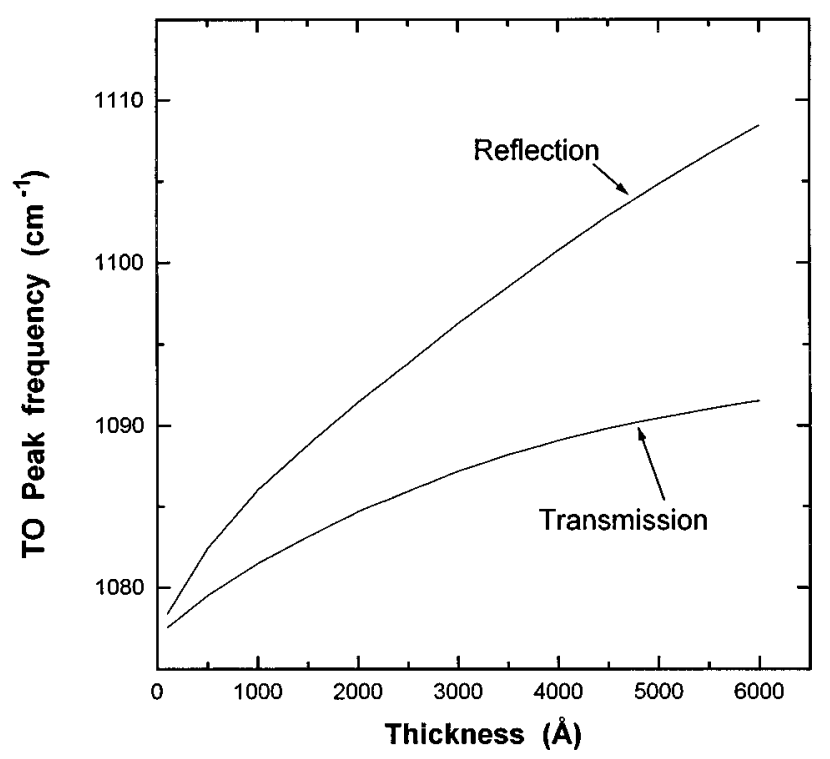

FIG. 3. Peak frequencies of the $\mathrm{TO}_{3}$ band of $\mathrm{SiO}_{2}$ on $\mathrm{Si}$ as a function of the thickness of the layer, for simulated reflection and transmission spectra.

peak frequency as a function of the thickness of the layer for both reflection and transmission (model 1). In transmission, $\mathrm{TO}_{3}$ peak frequency for $100 \AA$ thick samples is $1077 \mathrm{~cm}^{-1}$, but for $6000 \AA$ it is $1092 \mathrm{~cm}^{-1}$. These variations are similar to those mentioned in the introduction to the experimental measurements reported in the literature. In the same range of thicknesses, the width of the $\mathrm{TO}_{3}$ mode ranges between 67 and $82 \mathrm{~cm}^{-1}$. The evolution of the infrared band of $\mathrm{Si}_{3} \mathrm{~N}_{4}$ is similar, i.e., it shifts to higher frequencies as thickness increases. These results are compared with the experimental work presented in the next section.

Important modifications of the shape of the bands are also observed. For comparison, we have plotted the simulated absorption spectra of $\mathrm{a} \mathrm{SiO}_{2}$ layer of $100 \AA$ and another of $6500 \AA$ in Fig. 4; for comparison, the scales of the $y$ axis for the two plots were normalized so that the height of both

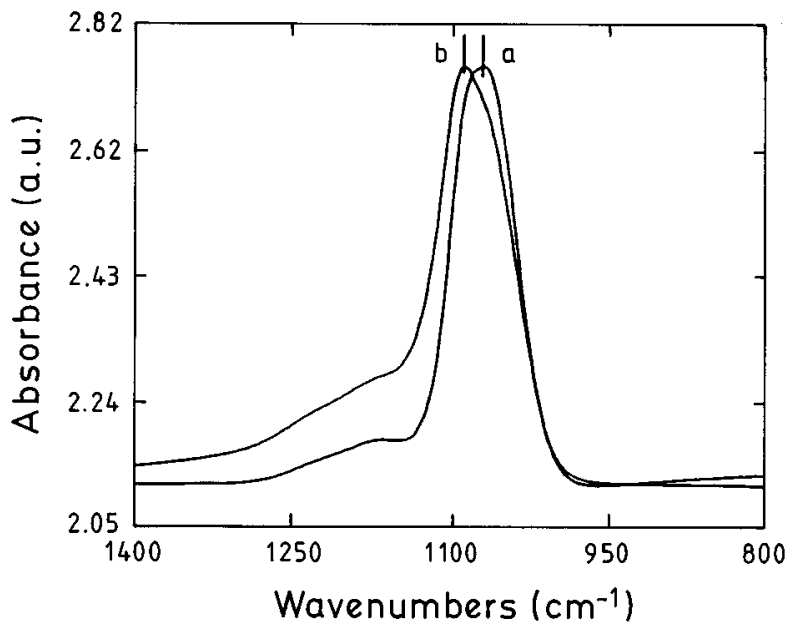

FIG. 4. Comparison between simulated absorption spectra at normal incidence of $\mathrm{SiO}_{2}$ on $\mathrm{Si}$ for different thicknesses: $100 \AA$ in (a), and $6500 \AA$ in (b). Both spectra are normalized to the same height for comparison of shape. 


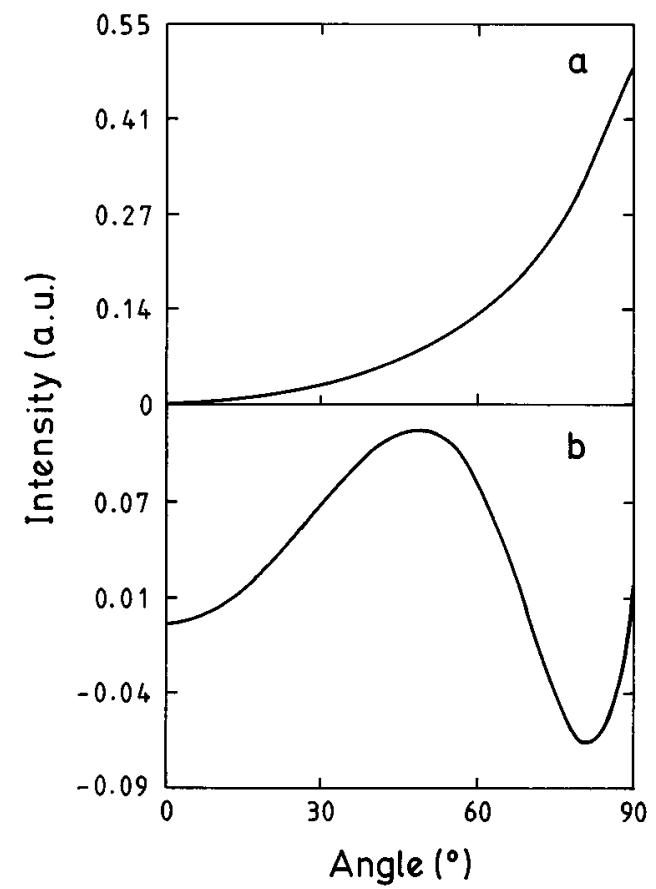

FIG. 5. (a) Intensity of the $\mathrm{LO}_{3}$ mode of $\mathrm{SiO}_{2}$ in absorbance $(-\log [$ transmittance $])$ as a function of the angle of incidence. (b) The same in reflection $(-\log [$ reflectance $])$.

peaks was the same. The frequency shift of the $\mathrm{TO}_{3}$ mode is evident, as is the greater high-frequency shoulder of the thicker sample $\left(\mathrm{TO}_{4}\right.$ band). Such dependence on thickness prevents us from indiscriminately analyzing the behavior of the $\mathrm{TO}_{4}$ band for quantifying disorder effects. The $\mathrm{TO}_{4}$ mode is nearly absent of the spectra of the very thin $\mathrm{SiO}_{2}$ layers (less than $50 \AA$ ). Lange ${ }^{6,9}$ did not find experimentally this shoulder for very thin samples, and other authors speculated about this fact. ${ }^{12,25,34}$ In the experimental spectra of very thin layers, superimposed to these effects there are obvious modifications of the structure, as the interface of $\mathrm{SiO}_{2}$ with the silicon substrate is far from being abrupt.

We found that the frequency and width dependence of the peaks on the angle of the incident radiation is not significant. In contrast, the intensities of both TO and LO peaks are strongly dependent on the angle of incidence in the following way. For $\mathrm{SiO}_{2}$ absorbance spectra, we found that the intensity of LO modes increased monotonically with the angle of incidence [Fig. 5(a)]. However, the behavior observed for reflection was unexpected: the LO intensity first increased, reached a maximum at approximately $45^{\circ}$, then decreased and changed in sign (negative peaks) between $60^{\circ}$ and $65^{\circ}$ [Fig. 5(b)]. This inversion in the sign of the LO mode was found around the Brewster angle for silicon $\left(72^{\circ}\right)$; at this value, the reflection coefficient of the $p$ wave at the silicon interface (which excites the LO mode) vanishes. Thus, large incident angles aid detection of LO bands (but far from the inversion point, see Fig. 5). However, the intensity of TO bands always decreased with the angle of incidence.

The dependence of the $\mathrm{TO}_{3}$ peak frequency on thickness for oblique incidence was similar to that for normal incidence. In contrast, irrespective of the angle of incidence

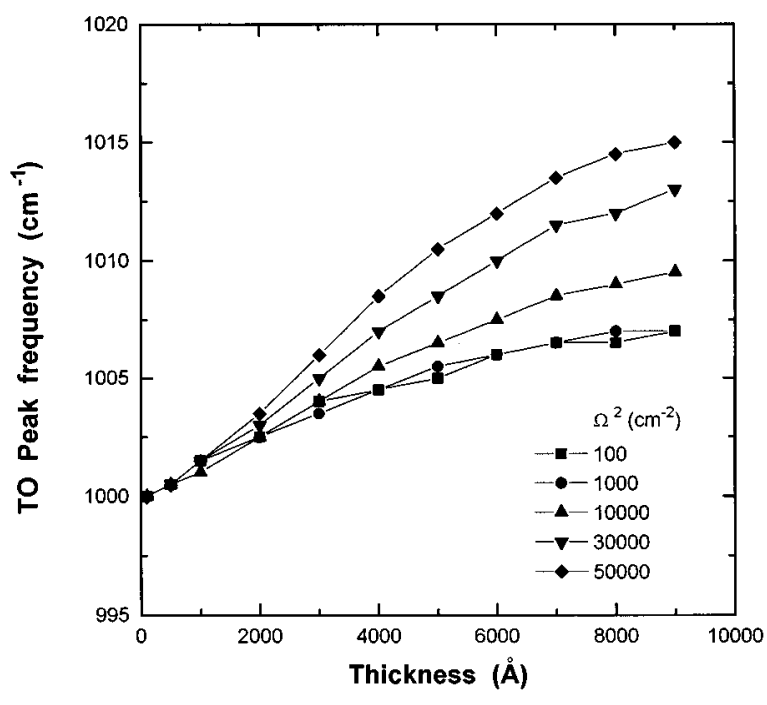

FIG. 6. TO peak frequency as a function of thickness for absorbance spectra at normal incidence, simulated from a dielectric function with a single mode (model 1). The curves are parametrized for different oscillator strengths $(\Omega)$ of the mode. The other parameters given as data for the simulation, where $\omega_{0}=1000 \mathrm{~cm}^{-1}$ and $\gamma=30$.

taken, the $\mathrm{LO}_{3}$ peak frequency changed only slightly (2-3 $\mathrm{cm}^{-1}$ ) within the whole range of thicknesses studied (100$6000 \AA$ A). Unfortunately, the $\mathrm{LO}_{3}$ mode also shows little variation with stress, composition, and microstructure. ${ }^{3,7,25}$

We also found similar spectral behavior for the simulated stretching bands of silicon nitride on silicon substrates. Peak frequencies and widths increased with thickness as for $\mathrm{SiO}_{2}$, so this behavior is expected to be quite general. Some experimental data on silicon nitrides are shown to correlate with the simulation results, as will be shown later.

With the aim of studying these geometrical effects on infrared bands in a more general way, several single bands (model 1) for different oscillator strengths were given as input data in to the simulation program. Then, the reflectance and transmittance spectra generated were analyzed as a function of the thickness of the layers. The most striking finding was remarkable differences in behavior depending on the oscillator strength. For strong bands, the behavior of peak frequency and width was similar to that reported above for $\mathrm{SiO}_{2}$, but for weak bands, for example, impurity bands such as the $\mathrm{Si}-\mathrm{H}$ or $\mathrm{N}-\mathrm{H}$ absorptions, the variation with thickness was less pronounced, and the asymptotic limit was much lower (Figs. 6 and 7).

Interference fringes for thick layers tend to distort spectral features, so a baseline spectral subtraction should be performed by reproducing the oscillating background. ${ }^{33}$ It can then be shown that the intensity of TO bands is linear with thickness in a wide range of thickness variation. In contrast, the intensity of the LO modes is linear for small thicknesses, but the slope of the curve changes (Fig. 8). Therefore, only the TO modes of the infrared spectra are suitable for thickness evaluation.

\section{EXPERIMENTAL PROCEDURE}

For the experiments, the samples used were 8000 and $2500 \AA$ thick silicon oxide layers on silicon $<100>$ wafers 


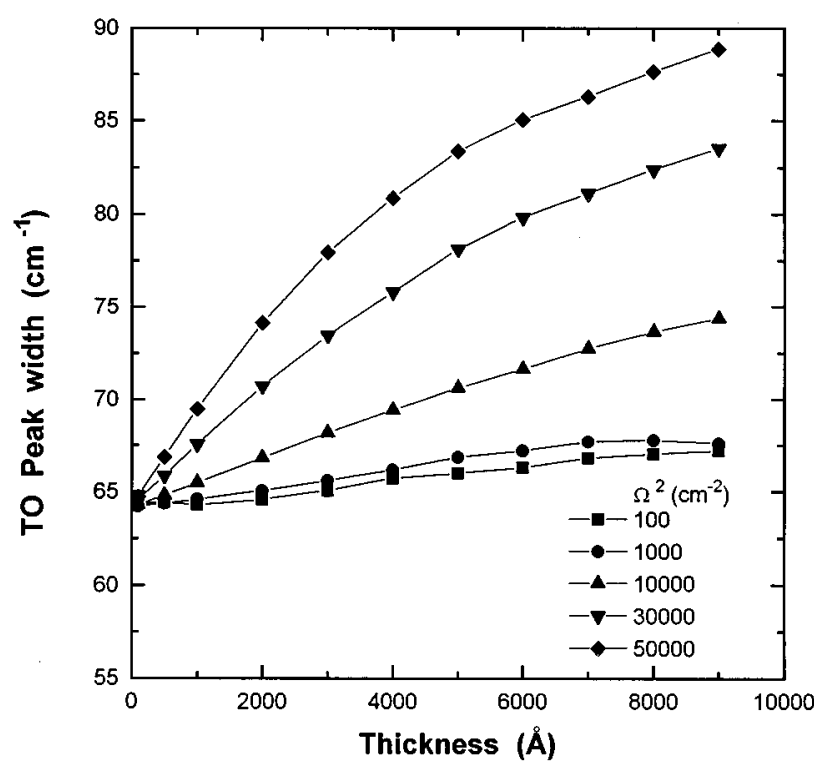

FIG. 7. TO peak width as a function of thickness for absorbance spectra at normal incidence, simulated from a dielectric function with a single mode (model 1). The curves are parametrized for different oscillator strengths $(\Omega)$ of the mode. The other parameters given as data for the simulation where $\omega_{0}=1000 \mathrm{~cm}^{-1}$ and $\gamma=30$.

with resistivity between 3 and $5 \Omega \mathrm{cm}$. The layers were grown by wet oxidation at $950{ }^{\circ} \mathrm{C}$ and annealed at $1100{ }^{\circ} \mathrm{C}$ in a rapid thermal annealing system for $10 \mathrm{~s}$. The other thicknesses were obtained after etching those layers in a buffered HF solution and measuring the remaining film thickness with optical interferometry by using a Nanospec AFT 200 of Nanometrics. For each etch step we averaged at least four thickness measurements taken at different points on the wafer. Before the infrared experiments, the oxide on the back of the wafers was etched off. The silicon nitride layers were ob-

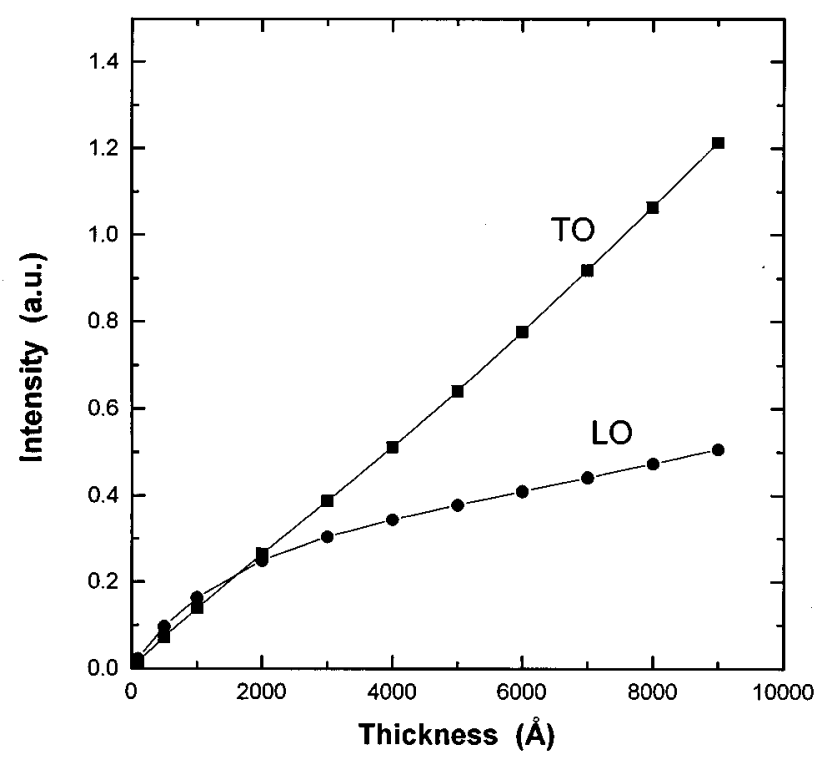

FIG. 8. Intensity of TO and LO modes as a function of thickness for simulated absorbance spectra at $45^{\circ}$ of incidence of $\mathrm{SiO}_{2}$ layers on $\mathrm{Si}$. It is clear that only TO modes are linear through the whole range of thicknesses studied.

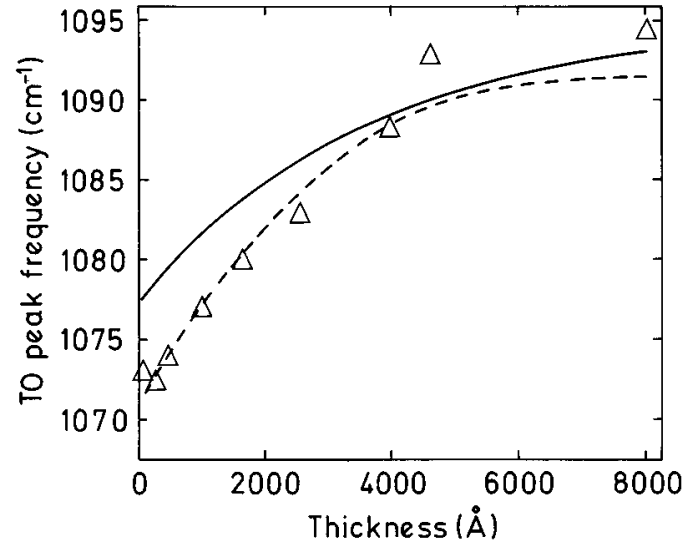

FIG. 9. Experimental $\mathrm{TO}_{3}$ peak frequency for absorbance at normal incidence as a function of the thickness of the $\mathrm{SiO}_{2}$ layer (triangles). Simulated behavior taking model 1 (full line) and the model 2 (broken line) for the dielectric function. Experimental data are very close to the predictions of model 2 .

tained in a low-pressure chemical vapor deposition system from $\mathrm{SiH}_{4}$ and $\mathrm{NH}_{3}$ as precursor gases. The deposition time was adjusted to obtain a suitable range of thicknesses, which were subsequently measured by ellipsometry.

The infrared measurements were performed with a BOMEM DA3 series Fourier transform infrared spectrophotometer with accessories to work in transmission and reflection at normal incidence or at incident angles between $20^{\circ}$ and $80^{\circ}$. The resolution limit was $1 \mathrm{~cm}^{-1}$ and at least 400 scans were averaged for each measurement. The infrared source was a globar, the beamsplitter of $\mathrm{KBr}$ and the detector was a high-sensitivity mercurium-cadmium-telluride (MCT) cooled by liquid nitrogen. From the single beam infrared spectra of the samples, we subtracted the spectra of the bare silicon references to eliminate silicon absorption components. Depending on layer thickness, an oscillating background was superimposed to the spectra (layer interferences), then a procedure to subtract this background was followed. ${ }^{33}$ As a result, flat base lines were accomplished for all the samples, irrespective of their thickness.

\section{EXPERIMENTAL RESULTS}

The experimental values of the peak frequencies of the $\mathrm{TO}_{3}$ silicon oxide absorption mode versus thickness are plotted in Fig. 9, together with the results of the simulation analysis. The full curve accounts for the results predicted from simulation by using model 1 . The dashed curve represents the results obtained from model 2. Model 2 closely reproduces the experimental results for the whole range of thicknesses, except for the two thicker samples (4600 and $8000 \AA$ ), where some differences occur. The origin of these differences will be discussed later. The results of the bandwidth for the $\mathrm{TO}_{3}$ mode are plotted in Fig. 10. Model 2 also reproduces the experimental results more closely, suggesting that it is more suitable for the analysis of amorphous materials. Furthermore, the shape of infrared bands obtained from model 2 better reproduced the experimental ones. The ex- 


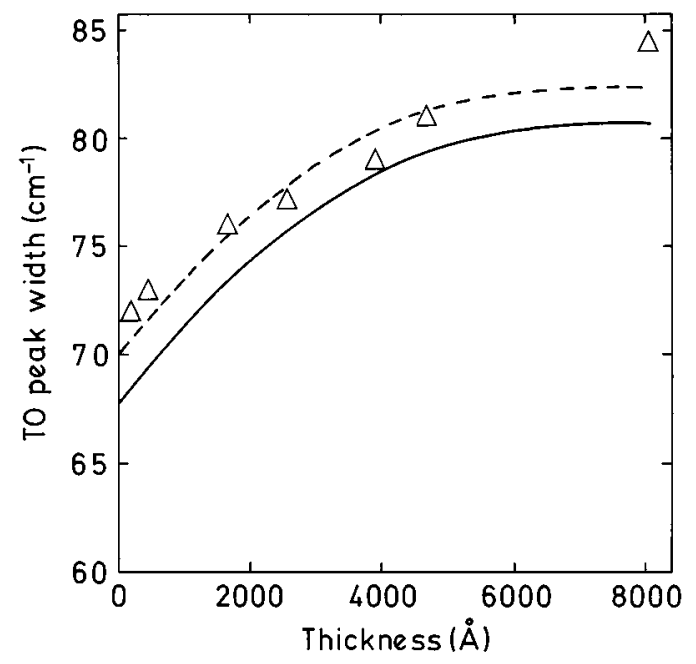

FIG. 10. Experimental $\mathrm{TO}_{3}$ peak width for absorbance at normal incidence as a function of the thickness of the $\mathrm{SiO}_{2}$ layer (triangles). Simulated behavior taking model 1 (full line) and the model 2 (broken line) for the dielectric function. Experimental data are very close to the predictions of model 2.

perimental peak frequencies for silicon nitride were also in agreement with the results of the simulations (Fig. 11).

The reflection spectra of $\mathrm{SiO}_{2}$ measured with different angles of incidence are plotted in Fig. 12. The most important effect is the band distortion and inversion of the LO peak at around $60^{\circ}$, which was predicted above. The experimental inversion of the $\mathrm{LO}$ peak in $\mathrm{SiO}_{2}$ at increasing angles of incidence was reported previously by other authors. ${ }^{35}$

\section{DISCUSSION}

In the previous sections we demonstrated, by both simulation and experiment, geometrical or optical effects in the study of the infrared spectra of multilayered systems. More-

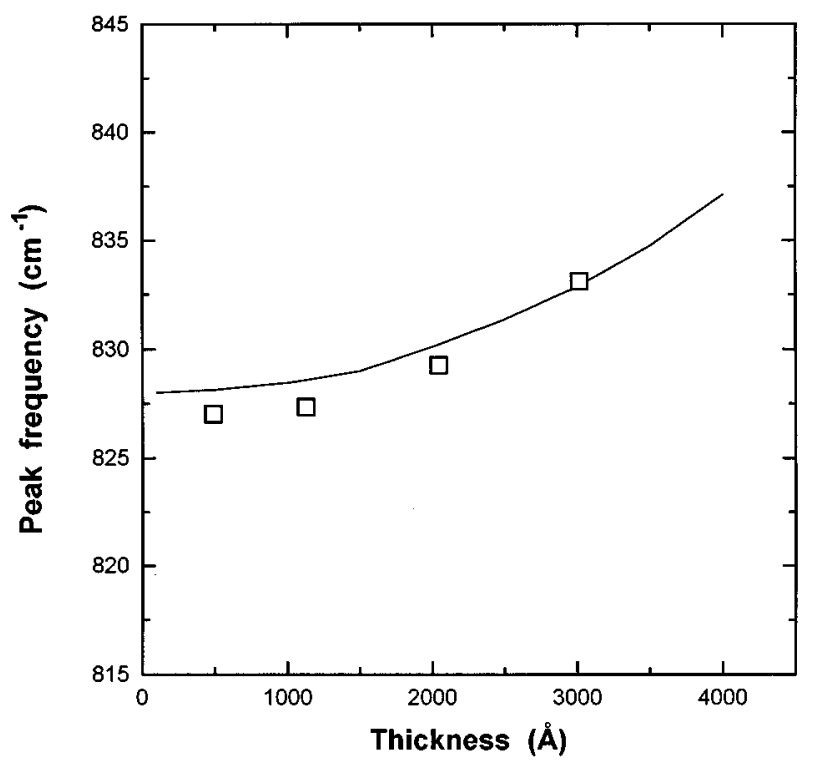

FIG. 11. Experimental silicon nitride peak frequency for absorbance at normal incidence as a function of the thickness of the layer (squares). The full line corresponds to the simulated results.

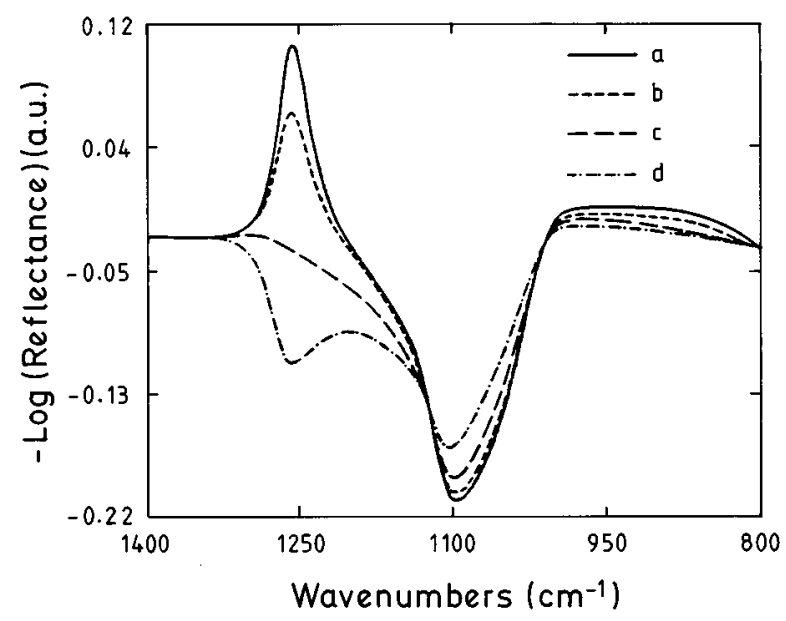

FIG. 12. Experimental reflection spectra of $\mathrm{SiO}_{2}$ layers obtained at different angles of incidence. (a) $30^{\circ}$, (b) $40^{\circ}$, (c) $50^{\circ}$, and (d) $60^{\circ}$. The inversion of the $\mathrm{LO}_{3}$ mode is clearly observed.

over, we showed that simulation and experiment correlate well in a wide range of thicknesses, in different materials, and for both reflection and transmission spectra. However, we have not discussed the origin of these effects, nor yet given any procedure to sort out this dilemma. These effects are because the absorption spectrum is not an intrinsic property of the material, as is the dielectric function. However, the calculation of frequency dependence of the dielectric function is difficult without sophisticated software and some previous knowledge of the optical constants.

The origin of these effects in layered systems is due to two main factors. First, we have to distinguish the problem of the boundaries and the interference effects of the partial beam intensities. The transformation of fields across the interface between two layers is given by the Fresnel coefficients, which depend on refractive indexes, extinction coefficients, and the angle of incidence. They give the fraction of the light that is transmitted or reflected across the boundary, and from their dependence they will mainly be influenced by the polarization and the angle of incidence of the incoming wave. Thus, polarization and angle effects on the spectra have their origins in the boundary conditions for the field across an interface. Then, the intensity of signal obtained from the TO and LO modes strongly depends on angles and polarization as we have reported above, predicting the distortion and inversion of the $\mathrm{LO}$ mode in reflection.

Alternatively, interference within the layers gives a sinusoidal base line dependence (Fabry-Perot resonances). Obviously, for multilayered systems, the equations governing the interferences are more complicated. The frequency of these background interferences is strongly dependent on the thickness of the layer (quicker interferences for thicker layers), which has a complicated effect on the peak frequency, width, and shape of the absorption bands. Nevertheless, it can be argued that if this sinusoidal base line could be reproduced exactly (for example, by means of a simulation program), then we would be able to subtract it from the experimental spectrum and so obtain the spectra without geometrical modifications. In fact, Maley ${ }^{33}$ proposed a 


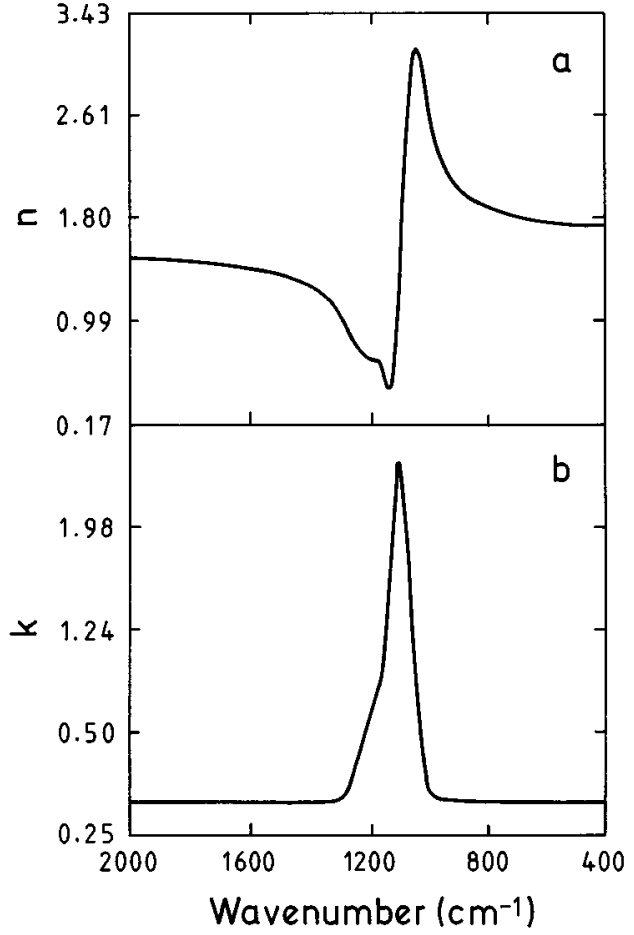

FIG. 13. (a) Real ( $n$ ) and (b) imaginary ( $k$ ) parts of the refractive index of silicon oxide from the parameters of Table I (model 2).

method to reproduce closely the sinusoidal interferences by setting the extinction coefficient $(k)$ equal to zero and optimizing the refractive index $(n)$ and the thickness $(d)$ of the layer in the exact equations of the multilayered-system. However, this is only approximate because the refractive index varies largely in the range of passing through absorbing bands, and then any constant parameter for fitting, even optimized, cannot reproduce the shape of the base line. Otherwise, to determine the variation of $n$ over the absorbing band requires the knowledge of the frequency dependence of the dielectric function, which is rather difficult, as we mentioned above. The total variation of the real part of the dielectric function through an absorbing band is connected with their respective oscillator strength values, $\Omega_{j}$, and is given by the sum rule

$$
\Omega_{j}^{2}=\frac{2}{\pi} \int_{\text {band } j} \nu \epsilon_{j}^{\prime \prime}(\nu) d \nu .
$$

As an illustration representing the variation of $n$ and $k$ through an absorbing band, we have plotted the real $(n)$ and imaginary $(k)$ parts of the complex refractive index of $\mathrm{SiO}_{2}$ in the frequency range of interest $\left(\mathrm{TO}_{3}-\mathrm{TO}_{4}\right.$ mode $)$ in Fig. 13. The calculations were performed by using the dielectric function generated from the parameters shown in Table I and taking into account that $(n+i k)^{2}=\epsilon$. Thus, any constant value for $n$ taken over this region can only give approximate results for reducing the degree of band deformations.

Nevertheless, shifts and distortions of the spectra also depend on the intensity of the absorption band. Strongly absorbing bands (i.e., high oscillator strength, $\Omega$ ) lead to higher deformations when the thickness increases. Thus, main infra- red bands for silicon oxide or silicon nitride, which are very strong, result in greater shifts and deformations than less intense bands such as impurity bands ( $\mathrm{Si}-\mathrm{H}, \mathrm{N}-\mathrm{H}$, etc.). Figures 6 and 7 show that the slope of the curves for low oscillator strengths is lower than that for intense bands, clearly indicating smaller shifts and deformations of the infrared spectra. The explanation of this behavior is also connected with the variation of $n$ over the absorbing band and, thus, with the modification of the base line. In fact, stronger bands have higher variations of $n$ in the absorbing region [Eq. (8)], and so, good base lines are more difficult to reproduce with only one constant value of $n$.

It is important to remark that we took into account coherent multiple reflections in the film and incoherent multiple reflections in the substrate. This is important since incoherence rules out the interference fringes. Therefore, as experiment and simulation gave very close results, this is a good choice for layers with thicknesses in the limit $d \ll \lambda$. However, for thicker layers (of the order of $\mu \mathrm{m}$ ) the thickness begins to be comparable to the wavelength (midinfrared range is $400-4000 \mathrm{~cm}^{-1}$ or $25-2.5 \mu \mathrm{m}$ ) and the behavior is neither coherent or incoherent. This is probably the reason why the experimental points of the two thicker layers of Fig. 9 do not reproduce the simulation behavior and deviate slightly from the simulated curve, which applies only at the coherent limit.

From the results presented above it is clear that some corrections must be taken into account for comparing the vibrational properties of samples with different thicknesses and/or different experimental setup. It is difficult to give only one expression for correcting these effects because the behavior depends on the oscillator strength of the absorption being studied and, thus, on the sample itself. As discussed above, the best procedure would be to obtain the dielectric function after fitting the experimental spectra. If this is not possible, the best choice is to extrapolate peak frequencies and widths of the infrared bands to the limit of zero thickness. The absorbance position when thickness tends to zero coincides with the maximum of the dielectric function. This is in accordance with the fact that for very thin layers, absorption spectra resembles the imaginary part of the dielectric function. ${ }^{25}$ Nevertheless, such extrapolation requires knowledge of the dependences on thickness, so simulation studies should be performed at least once to obtain the desired dependence for a given material. If simulation is not available, the general dependences versus thickness given in Fig. 6 can be used, provided the oscillator strength of the absorption is known.

We have fitted the peak frequency of $\mathrm{SiO}_{2}$ and $\mathrm{Si}_{3} \mathrm{~N}_{4}$ layers with quadratic polynomials in $d$ (error less than $3 \%$ ). The corrections to zero thickness can be performed by subtracting from the experimental values the calculated values of the terms in Eq. (9) or Eq. (10) that depend on $d$ (i.e., the sum of the linear and quadratic terms). The following equations are valid for transmission at normal incidence in a wide range of thicknesses ( $d$ in $\mu \mathrm{m}$ and valid up to $1 \mu \mathrm{m}$ ):

$$
\begin{aligned}
\omega_{\max }\left(\mathrm{SiO}_{2}\right)= & 1070.98+65.07 d-5.36 \times 10^{-3} d^{2} \\
& \left(\mathrm{~cm}^{-1}\right),
\end{aligned}
$$




$$
\omega_{\max }\left(\mathrm{Si}_{3} \mathrm{~N}_{4}\right)=830.60+11.41 d+4.16 \times 10^{-3} d^{2}
$$

$$
\left(\mathrm{cm}^{-1}\right) \text {. }
$$

These corrections were successfully applied by us to evaluate the infrared data in analyzing thin silicon oxides, ${ }^{7}$ in the study of implanted oxides, ${ }^{8,25}$ and in the analysis of buried oxides, where we found pronounced effects depending on the depth of the buried layer and the size in general. ${ }^{23,24}$

\section{ANALYTICAL APPROXIMATIONS FOR THE REFLECTANCE AND TRANSMITTANCE OF VERY THIN FILMS}

Some of the previous results can be clearly understood if analytical approximations for the multilayer optics are developed. The formulae for the transmission and reflection are complex even for a system composed of a thin layer and a substrate. Many authors proposed analytically exact or approximate formulae for a single layer on a substrate under normal or oblique incidence. ${ }^{36,37}$ Developing approximate expressions giving the explicit dependence of transmission and reflection on the thickness of the layer is useful for our purposes.

After developing Eqs. 1-3 for only one layer on a substrate, the transmission $(t)$ and reflection $(r)$ coefficients of the system, respectively, take the following form: ${ }^{27}$

$$
\begin{aligned}
& t=\frac{2 \cos \alpha}{\left(\cos \alpha+\eta_{b}\right) \cos N \delta-i\left(\eta+\eta_{b} \cos \alpha / \eta\right) \sin N \delta} \\
& r=\frac{\left(\cos \alpha-\eta_{b}\right) \cos N \delta+i\left(\eta-\eta_{b} \cos \alpha / \eta\right) \sin N \delta}{\left(\cos \alpha+\eta_{b}\right) \cos N \delta-i\left(\eta+\eta_{b} \cos \alpha / \eta\right) \sin N \delta}
\end{aligned}
$$

where the subscript $b$ stands for the substrate, $\delta=2 \pi d / \lambda$ is the optical path inside the layer, $N$ is the generalized refractive index of the layer, $\alpha$ is the angle of incidence, and $\eta=N$ for $s$ polarization or $\eta=N / \epsilon$ for $p$ polarization. The reflection $(R)$ and transmission $(T)$ of the system are calculated from the coefficients in Eqs. (11) and (12) by using Eqs. (4) and (5), and result in complicated expressions dependent on thicknesses, refractive indexes, angle, and dielectric functions of the layer and the substrate.

We discuss the expressions for first and second order in $\delta$, which are obtained after developing the equations of $T$ and $R$ in Taylor series. The result of these approximations for first order (very thin layers compared with wavelength) are given by the following equations:

$$
\begin{aligned}
& T_{\mathrm{pol} s}=\frac{4 N_{b} \cos \alpha}{\left(\cos \alpha+N_{b}\right)^{2}}\left\{1-\frac{2 \operatorname{Im}(\epsilon)}{\cos \alpha+N_{b}} \delta\right\}, \\
& T_{\mathrm{pol} p}=\frac{4 N_{b} \cos \alpha}{\left(\cos \alpha+N_{b} / \epsilon_{b}\right)^{2}}\left\{1-\frac{2\left[\operatorname{Im}(-1 / \epsilon) \sin ^{2} \alpha+\operatorname{Im}(\epsilon)\left(N_{b} / \epsilon_{b}\right) \cos \alpha\right]}{\cos \alpha+N_{b} / \epsilon_{b}} \delta\right\}, \\
& R_{\mathrm{pol} s}=\left(\frac{\cos \alpha-N_{b}}{\cos \alpha+N_{b}}\right)^{2}\left\{1+\frac{4 \cos \alpha \operatorname{Im}(\epsilon)}{\epsilon_{b}-1} \delta\right\}, \\
& R_{\mathrm{pol} p}=\left(\frac{\cos \alpha-N_{b} / \epsilon_{b}}{\cos \alpha+N_{b} / \epsilon_{b}}\right)^{2}\left\{1-4 \cos \alpha \frac{\left[-\operatorname{Im}(-1 / \epsilon) \sin ^{2} \alpha+\left(N_{b} / \epsilon_{b}\right)^{2} \operatorname{Im}(\epsilon) \cos ^{2} \alpha\right]}{\cos ^{2} \alpha-\left(N_{b} / \epsilon_{b}\right)^{2}} \delta\right\} .
\end{aligned}
$$

The transmission and reflection of the layer for $s$ polarization $\left(T_{\text {pol } s}\right.$ and $\left.R_{\text {pol } s}\right)$ take, respectively, their minimum and maximum values (absorption peaks) at a maximum value of the imaginary part of the dielectric function, $\operatorname{Im}(\epsilon)$, i.e., at the poles of the dielectric function that are related to the TO modes. However, for $p$ polarization there are additional terms dependent on $\operatorname{Im}(-1 / \epsilon)$ so, other maxima or minima occur in $T_{\text {pol } p}$ and $R_{\text {pol } p}$ when $\operatorname{Im}(-1 / \epsilon)$ is in its maximum, i.e., at the zeros of the complex dielectric function that are related to the LO modes. ${ }^{5,27,32}$ Both LO and TO modes are affected by angular factors. If, for simplicity, we take the substrate as the vacuum, the angular dependence of the intensity of the LO and TO modes in $T_{\text {pol } p}$ is TO $\propto \cos \alpha$ and $\mathrm{LO} \propto(\sin \alpha)^{2} / \cos \alpha$. Therefore, for normal incidence the intensity of LO modes is zero. Furthermore, by analyzing the angular dependence of the LO modes in $R_{\text {pol } p}$ [Eq. (16)], it is easy to find that the term responsible for the inversion of the LO modes is $\left(\cos \alpha-N_{b} / \epsilon_{b}\right)$, which changes sign at the Brewster angle of the substrate; so, the inversion of the LO modes and the angular dependence of TO and LO modes arise as a consequence of the substrate refractive index and are independent of the thickness and refractive index of the layer for very thin layers. This is in agreement with the results of the simulation and the experimental results reported above.

The transmittance for $s$ polarization [Eq. (13)] depend linearly on $\operatorname{Im}(\epsilon)$ and then the transmission spectra of thin layers are similar to the frequency dependence of the imaginary part of the dielectric function. So, we can use these expressions to obtain approximate values of $\operatorname{Im}(\epsilon)$ at the limit of very thin layers. If we refer to the quotient between $T_{\text {pol } s}$ and the common factor in Eq. (13) (transmittance of the substrate) as $T_{\mathrm{RS}}$, then 


$$
\epsilon^{\prime \prime}=\operatorname{Im}(\epsilon)=\frac{\left(1-T_{\mathrm{RS}}\right)\left(\cos \alpha+N_{b}\right)}{2 \delta} .
$$

The dielectric function in the infrared range can be obtained by this method within an error of $10 \%$ in a range of thicknesses up to $500 \AA$. With the aim of evaluating secondorder effects, we also developed the formulae for the $\delta^{2}$ term. The results obtained for transmission under $s$ and $p$ polarization are

$$
\begin{aligned}
T_{\mathrm{pol} s}= & \frac{4 \cos \alpha N_{b}}{\left(\cos \alpha+N_{b}\right)^{2}}\left\{1-\frac{2 \operatorname{Im}(\epsilon)}{\cos \alpha+N_{b}} \delta+\frac{3[\operatorname{Im}(\epsilon)]^{2}-[\operatorname{Re}(\epsilon)]^{2}-\left(1+\epsilon_{b}\right) \operatorname{Re}(\epsilon)+\epsilon_{b}}{\left(\cos \alpha+N_{b}\right)^{2}} \delta^{2}\right\}, \\
T_{\mathrm{pol} p}= & \frac{4 \cos \alpha N_{b}}{\left(\cos \alpha+\frac{N_{b}}{\epsilon_{b}}\right)^{2}}\left\{1-\frac{2\left[\operatorname{Im}(-1 / \epsilon) \sin ^{2} \alpha+\operatorname{Im}(\epsilon)\left(N_{b} / \epsilon_{b}\right) \cos \alpha\right]}{\cos \alpha+N_{b} / \epsilon_{b}} \delta\right. \\
& \left.+\frac{\left\{3[\operatorname{Im}(\epsilon)]^{2}-\operatorname{Re}(\epsilon)\right\}-\left[\left(N_{b} / \epsilon_{b}\right) \cos \alpha+\sin ^{2} \alpha /|\epsilon|^{2}\right]+\operatorname{Re}(\epsilon)\left(\cos ^{2} \alpha+N_{b}^{2} / \epsilon_{b}^{2}+2 \sin ^{2} \alpha /|\epsilon|^{2}\right)}{\left(\cos \alpha+N_{b} / \epsilon_{b}\right)^{2}} \delta^{2}\right\} .
\end{aligned}
$$

Finally, in order to verify the validity of the approximations, we applied the above equations to the simulated transmission and reflection spectra of a $\mathrm{SiO}_{2}$ layer of $500 \AA$. The equations under the second-order approximation depend on both $\operatorname{Re}[\epsilon]$ and $\operatorname{Im}[\epsilon]$ and, thus, we need to know both $T$ and $R$. Then, two different types of dielectric function obtained from the first order (only for transmittance) and from the second order (for both transmittance and reflectance) equations were compared with the dielectric function used as data for the simulation. The results are plotted in Fig. 14. The second-order approximations can give dielectric functions for a $500 \AA$ layer with a relative error of less than $5 \%$.

\section{SUMMARY AND CONCLUSIONS}

The geometrical or optical modifications of infrared multilayer spectra with dimensions, arrangements, angle, and polarization have been studied both by simulation and ex-

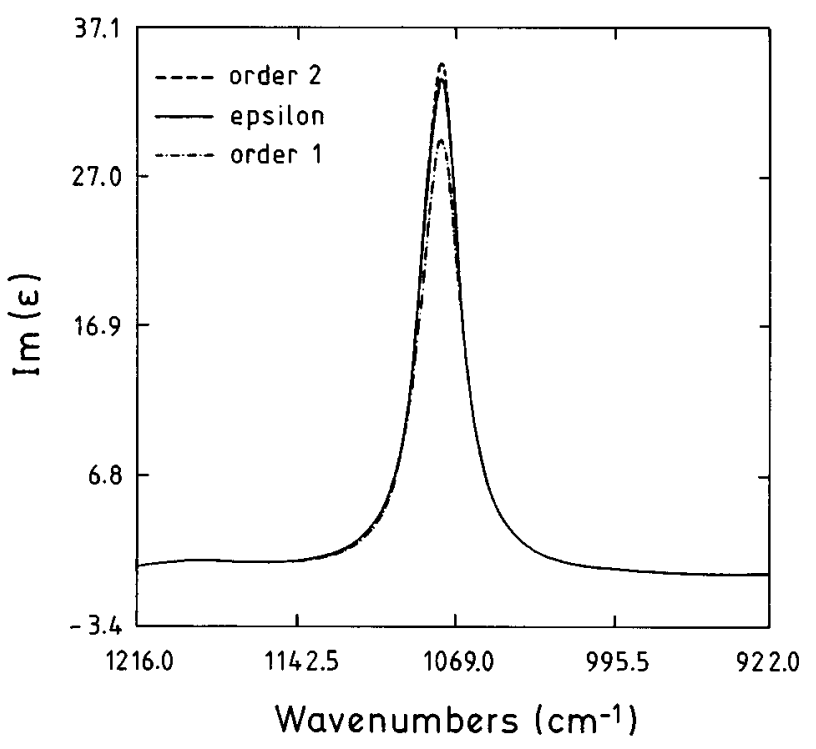

FIG. 14. Imaginary part of the dielectric function of a single peak and the results obtained from the first- and second-order approximation of the transmittance and reflectance of a thin layer of $500 \AA$ thick. perimentally. Simulated and experimental results of position and width of TO bands versus thickness of the layer were obtained for silicon oxide and silicon nitride. Also, the results of model calculations for individual peaks with different oscillator strength values are shown. Peak frequency and width always increase with thickness, and the rate of change also increases with the magnitude of the oscillator strength. For thicknesses near $1 \mu \mathrm{m}$, these frequency and width values saturated. In contrast, LO bands are not modified in the whole range of thicknesses. Moreover, the angle dependence of the intensity and position of LO and TO modes were studied experimentally and by simulation. The theoretically predicted inversion of the LO mode of silicon oxide in reflection at approximately $60^{\circ}$ of incidence was also experimentally verified.

The origin of these effects is discussed, separating the influence of the boundaries and the interference effects of the partial beam intensities. The dependence on the oscillator strength is explained in terms of a large variation of the refractive index when the frequency passes through a strongly absorbing band. Possible ways of applying corrections to the experimental spectra and some examples are also shown and discussed. Finally, analytical approximations for the reflectance and transmittance of thin layers are developed as a function of the thickness of the layer. The main results of the evolution of $\mathrm{TO}$ and LO modes are deduced from these approximations. First- and second-order approximations allowed us to obtain the imaginary part of the dielectric function from only transmittance and from both transmittance and reflectance, respectively, measurements for thin layers.

\section{APPENDIX}

We consider here the coordinates and angles defined for the multilayer plotted in Fig. 1. The matrix for the transformation of fields across the interface $z_{j}$ between two layers $j$ and $j-1$, takes the form

$$
\left[\mathbf{M}_{j}\right]=\left(\begin{array}{cc}
\tau_{j, j-1}^{-1} & \rho_{j, j-1} \tau_{j, j-1}^{-1} \\
\rho_{j, j-1} \tau_{j, j-1}^{-1} & \tau_{j, j-1}^{-1}
\end{array}\right),
$$


where $\rho_{j, j-1}$ and $\tau_{j, j-1}$ are Fresnel's complex-amplitude reflection and transmission coefficients. For $s$ polarization (electric field parallel to interfaces),

$$
\begin{gathered}
\rho_{j, j-1}=\frac{N_{j}-N_{j-1}}{N_{j}+N_{j-1}}, \\
\tau_{j, j-1}=\frac{2 N_{j}}{N_{j}+N_{j-1}},
\end{gathered}
$$

where $N_{j}$ is the generalized refractive index of the $j$ th layer. The use of $N_{j}$ instead of the complex refractive index $n_{j}$ allows us to write all the equations in the same way as for normal incidence. It is defined as

$$
N_{j}=\sqrt{\epsilon_{j}-\sin ^{2}(\alpha)},
$$

where $\epsilon_{j}$ is the dielectric function of the $j$ th layer and $\alpha$ is the angle of incidence. The matrix for the transformation through a layer $j$, from right to left, is

$$
\left.\left[P_{j}\right]=\left(\begin{array}{cc}
\exp \left(-i \frac{\omega}{c} N_{j} d_{j}\right. & \exp \left(i \frac{\omega}{c} N_{j} d_{j}\right.
\end{array}\right)\right)
$$

where $d_{j}$ is the thickness of the $j$ th layer.

In the case of $p$ polarization, the magnetic field is parallel to the interfaces. Then, we have to consider the incident, reflected, and transmitted fields $\mathbf{H}_{i}, \mathbf{H}_{r}$, and $\mathbf{H}_{t}$, respectively, instead of the electric fields in Eq. (1). Fresnel's coefficients are given by

$$
\begin{gathered}
\rho_{j, j-1}=\frac{N_{j} / \epsilon_{j}-N_{j-1} / \epsilon_{j-1}}{N_{j} / \epsilon_{j}+N_{j-1} / \epsilon_{j-1}}, \\
\tau_{j, j-1}=\frac{2 N_{j} / \epsilon_{j}}{N_{j} / \epsilon_{j}+N_{j-1} / \epsilon_{j-1}} .
\end{gathered}
$$

Finally, the factor $\operatorname{Re}\left[N_{b}\right]$ in Eq. (5) must be changed to $\operatorname{Re}\left[N_{b} / \epsilon_{b}\right]$.

${ }^{1}$ J. T. Fitch, G. Lucosky, E. Kobeda, and E. A. Irene, J. Vac. Sci. Technol. B 7, 153 (1988).

${ }^{2}$ J. T. Fitch, C. H. Bjorkman, G. Lucosky, F. H. Pollak, and X. Yin, J. Vac. Sci. Technol. B 7, 775 (1989).

${ }^{3}$ A. Lehmann, L. Schumann, and K. Hubner, Phys. Status Solidi 117, 689 (1983).
${ }^{4}$ M. L. Naiman, C. T. Kirk, B. L. Emerson, and J. B. Taitel, J. Appl. Phys. 58, 779 (1985).

${ }^{5}$ C. T. Kirk, Phys. Rev. B 38, 1255 (1988).

${ }^{6}$ P. Lange, J. Appl. Phys. 66, 201 (1989).

${ }^{7}$ B. Garrido, J. Samitier, J. R. Morante, L. Fonseca, and F. Campabadal, Appl. Surf. Sci. 56-58, 861 (1992).

${ }^{8}$ B. Garrido, C. Domínguez, J. Montserrat, J. R. Morante, and J. Samitier, Phys. Rev. B 49, 14845 (1994).

${ }^{9}$ P. Lange, U. Schnakenberg, S. Ullerich, and H. J. Schliwinski, J. Appl. Phys. 68, 3532 (1990).

${ }^{10}$ F. J. Grunthaner and P. J. Grunthaner, Mater. Sci. Rep. 1, 65 (1986).

${ }^{11}$ G. Hollinger and F. J. Himpsel, Appl. Phys. Lett. 44, 93 (1984).

${ }^{12}$ I. W. Boyd and J. I. B. Wilson, J. Appl. Phys. 62, 3195 (1987).

${ }^{13}$ R. A. B. Devine, Trans. Mater. Res. Soc. Jpn. 8, 165 (1992).

${ }^{14}$ R. A. B. Devine, J. Electron. Mater. 19, 1299 (1990).

${ }^{15}$ R. M. Almeida and C. G. Pantano, J. Appl. Phys. 68, 4225 (1990).

${ }^{16}$ F. Stolze, M. Zacharias, S. Schippel, and B. Garke, Solid State Commun. 87, 805 (1993).

${ }^{17}$ M. Nakamura, Y. Mochizuki, K. Usami, Y. Itoh, and T. Nozaki, Solid State Commun. 50, 1079 (1984).

${ }^{18}$ W. A. Lanford and M. J. Rand, J. Appl. Phys. 49, 2473 (1978).

${ }^{19}$ I. Kato, K. Noguchi, and K. Numada, J. Appl. Phys. 62, 492 (1987).

${ }^{20}$ C. J. Fang, K. J. Gruntz, L. Ley, and M. Cardona, J. Non-Cryst. Solids 35\&36, 255 (1980).

${ }^{21}$ G. Lucovsky, J. Yang, S. S. Chao, J. E. Tyler, and W. Czubatyj, Phys. Rev. B 28, 3225 (1983).

${ }^{22}$ M. H. Brodsky, M. Cardona, and J. J. Cuomo, Phys. Rev. B 16, 3556 (1977).

${ }^{23}$ A. Pérez, J. Samitier, A. Cornet, J. R. Morante, P. L. F. Hemment, and K. P. Homewood, Appl. Phys. Lett. 57, 2433 (1990).

${ }^{24}$ J. Samitier, S. Martínez, A. Pérez-Rodríguez, B. Garrido, J. R. Morante, A. M. Papon, and J. Margail, Nucl. Instrum. Methods Phys. Res. B 80/81, 838 (1993) (see Ref. 23).

${ }^{25}$ B. Garrido, doctoral thesis, University of Barcelona, 1993.

${ }^{26}$ C. Martinet and R. A. B. Devine, J. Appl. Phys. 77, (1995).

${ }^{27}$ B. Harbecke, Appl. Phys. B 39, 165 (1986).

${ }^{28} \mathrm{~F}$. Abelès, Advanced Optical Techniques (North Holland, Amsterdam, 1967).

${ }^{29}$ Z. Knittl, Optics of Thin Films (Wiley, London, 1976).

${ }^{30}$ P. Grosse, B. Harbecke, B. Heinz, and R. Meyer, Appl. Phys. A 39, 257 (1986).

${ }^{31}$ I. P. Lisovskii, V. G. Litovhenko, V. G. Lozinskii, and G. I. Steblovskii, Thin Solid Films 213, 164 (1992).

${ }^{32}$ D. W. Berreman, Phys. Rev. 130, 2193 (1963).

${ }^{33}$ N. Maley, Phys. Rev. B 46, 2078 (1992).

${ }^{34}$ Y. S. Yen and J. S. Wong, Mikrochimica Acta 1, 441 (1988).

${ }^{35}$ L. Vriens and W. Ripens, Appl. Opt. 22, 4105 (1983).

${ }^{36}$ S. G. Tomlin, J. Phys. D 5, 847 (1972).

${ }^{37}$ B. Garrido, J. A. Moreno, J. Samitier, and J. R. Morante, Appl. Surf. Sci. 63, 236 (1993). 